\title{
Photoluminescence studies of rare earth (Er, Eu, Tm) in situ doped $\mathrm{GaN}$
}

\author{
U. Hömmerich $^{\text {a,* }}{ }^{\text {, Ei Ei Nyein }}{ }^{\text {a }}$, D.S. Lee ${ }^{\text {b }}$, J. Heikenfeld ${ }^{\text {b }}$, A.J. Steckl ${ }^{\text {b }}$, J.M. Zavada ${ }^{c}$ \\ a Department of Physics, Hampton University, Hampton, VA 23668, USA \\ b Nanoelectronics Laboratory, University of Cincinnati, Cincinnati, OH 45221, USA \\ c US Army Research Office, Durham, NC 27709, USA
}

\begin{abstract}
The emission properties of rare earth (RE)-doped GaN are of significant current interest for applications in full color displays, white lighting technology, and optical communications. We are currently investigating the photoluminescence (PL) properties of RE (Er, Eu, Tm)-doped $\mathrm{GaN}$ thin-films prepared by solid-source molecular beam epitaxy. The most intense visible PL under above-gap excitation is observed from $\mathrm{GaN}: \mathrm{Eu}$ (red: $622 \mathrm{~nm}$ ) followed by GaN:Er (green: $537 \mathrm{~nm}, 558 \mathrm{~nm}$ ), and then GaN:Tm (blue: $479 \mathrm{~nm}$ ). In this paper, we present spectroscopic results on the Ga-flux dependence of the $\mathrm{Er}^{3+} \mathrm{PL}$ properties from $\mathrm{GaN}: \mathrm{Er}$ and we report on the identification of different $\mathrm{Eu}^{3+}$ centers in $\mathrm{GaN}$ :Eu through high-resolution PL excitation (PLE) studies. In addition, we observed an enhancement of the blue Tm ${ }^{3+} \mathrm{PL}$ from AlGaN:Tm compared to $\mathrm{GaN}$ :Tm. Intense blue PL from $\mathrm{Tm}^{3+}$ ions was also obtained from AlN:Tm under below-gap pumping.
\end{abstract}

(C) 2003 Elsevier B.V. All rights reserved.

PACS: 78.40. Fy; 78.55.-m; 78.55.Et

Keywords: Rare earth; GaN; AlGaN; Luminescence

\section{Introduction}

Rare earth (RE)-doped III-nitrides have recently emerged as a new class of phosphor materials for thin- and thick-film electroluminescence devices [1-4]. Compared to previously studied RE-doped semiconductors with relatively small band-gap $(<\sim 1.5 \mathrm{eV})$ like e.g. GaAs or Si [5], RE doping of wide band-gap semiconductors such as GaN, AlN, and $\mathrm{SiC}$ has led to the observation of intense RE emission at room temperature [6,7]. In addition, studies of GaN:Er and $\mathrm{GaN}: \mathrm{Eu}$ have shown that $\mathrm{RE}^{3+}$ ions can be incorporated into $\mathrm{GaN}$ at concentrations as high as $1-2$ at.\% without significant emission concentration quenching [8,9]. Current research efforts on RE-doped nitrides are focused on the optimization of existing materials and EL devices [2,3,7], evaluation of different doping techniques and dopant/host combinations $[4,7,10-12]$, as well as fundamental spectroscopic studies aimed towards a better understanding of the RE incorporation, excitation schemes, and emission efficiency [7,13-16].

\footnotetext{
* Corresponding author. Tel.: +1-757-727-5829; fax: +1-757-728-6910.

E-mail address: uwe.hommerich@hamptonu.edu (U. Hömmerich).
}

In this paper, we present spectroscopic results of the PL properties of GaN:Er as a function of Ga-flux employed during molecular beam epitaxy (MBE) growth. As will be discussed in more detail, the Ga-flux during growth significantly impacts the $\mathrm{Er}^{3+}$ lattice location and the concentration of optically active $\mathrm{Er}^{3+}$ ions. High-resolution PL excitation (PLE) studies were carried out on GaN:Eu, which allowed the identification of ${ }^{5} \mathrm{D}_{0} \leftrightarrow{ }^{7} \mathrm{~F}_{0}$ transitions and associated $\mathrm{Eu}^{3+}$ centers. Finally, we report on initial PL studies of Tm-doped $\mathrm{Al}_{x} \mathrm{Ga}_{1-x} \mathrm{~N}(0 \leq x \leq 1)$ and the enhancement of the blue $\mathrm{Tm}^{3+}$ emission with increasing $\mathrm{Al}$ content.

\section{Experimental details}

Rare earth (Er, Eu, Tm)-doped $\mathrm{GaN}$ and Tm-doped $\mathrm{Al}_{x} \mathrm{Ga}_{1-x} \mathrm{~N}$ films with $x=0.13,0.24,0.29,0.44$, and 1 (AlN) were grown by solid-source MBE on p-type $\operatorname{Si}\left(\begin{array}{lll}1 & 1 & 1\end{array}\right)$ substrates. Elemental $\mathrm{Ga}, \mathrm{Al}$, and RE sources were used in conjunction with a radio frequency (rf)-plasma source supplying atomic nitrogen. The RE concentration in the $\mathrm{GaN}$ and AlGaN films varied between $\sim 0.5$ and $\sim 1$ at. $\%$. More details on the sample preparation were published previously $[2,17]$. 
PL spectra were measured using either the UV argon laser lines $(336-363 \mathrm{~nm})$ or a visible argon laser line at $496.5 \mathrm{~nm}$. PL excitation spectra were recorded using an Optical Parametric Oscillator system as the excitation source. For low-temperature PL measurements the samples were mounted on the cold-finger of a closed-cycle helium refrigerator. Infrared PL spectra were recorded using a $1 \mathrm{~m}$ monochromator equipped with a liquid-nitrogen cooled $\mathrm{Ge}$ detector. In visible PL studies a thermo-electric cooled photomultiplier tube was employed for detection. The signal was processed using lock-in techniques or using a boxcar averager. The obtained PL spectra were not corrected for the spectral response of the setup.

\section{Results and discussion}

\subsection{Er-doped GaN}

It was previously reported that the visible and infrared emission properties of $\mathrm{Er}^{3+}$ ions in GaN films are strongly dependent on the Ga-flux during growth [18]. Under above band-gap excitation the $\mathrm{Er}^{3+}$ PL emission reached its maximum under slightly N-rich growth conditions. On the contrary, the band-edge emission from the GaN host reached its maximum under Ga-rich growth. This observation lead to the conclusion that the Ga-flux strongly influences the carrier-mediated excitation efficiency of $\mathrm{Er}^{3+}$ ions [18]. In the following, more detailed spectroscopic study on the Ga-flux dependence of the $\mathrm{Er}^{3+}$ PL properties are presented. Er-doped GaN samples grown under Ga-fluxes with beam equivalent pressures ranging from $2.2 \times 10^{-7}$ Torr ("N-rich") to $6.9 \times 10^{-7}$ Torr ("Ga-rich") were investigated [18]. The stoichiometric growth condition as determined by film thickness saturation was $\sim 4$ to $5 \times 10^{-7}$ Torr.

The visible and IR PL spectra under below band-gap excitation $(496.5 \mathrm{~nm})$ are shown in Fig. 1. This excitation wavelength overlaps an intra-4f $\mathrm{Er}^{3+}$ absorption line $\left({ }^{4} \mathrm{I}_{15 / 2} \rightarrow\right.$ $\left.{ }^{4} \mathrm{~F}_{9 / 2}\right)[19,20]$. Similar to above-gap excitation [18], $\mathrm{the}^{\mathrm{Er}} \mathrm{r}^{3+}$ PL strongly varied with Ga-flux under resonant excitation and reached a maximum under slightly $\mathrm{N}$-rich growth condition. Since both excitation schemes, carried-mediated and resonant intra-4f, exhibited the same $\mathrm{Er}^{3+} \mathrm{PL}$ behavior it can be concluded that the carrier-mediated $\mathrm{Er}^{3+}$ excitation is only weakly affected by the Ga-flux during growth.

Fig. 2 shows the high-resolution $1.54 \mu \mathrm{m} \mathrm{Er}^{3+}$ PL spectra of GaN:Er samples grown under different Ga-fluxes. A significant decrease in the $1.54 \mu \mathrm{m}$ PL linewidth can be noticed as well as the appearance of new spectral features with increasing Ga-flux. The spectral narrowing of the emission lines is consistent with the higher crystalline quality of $\mathrm{GaN}$ grown under Ga-rich conditions [18,21]. The observation of new spectral lines indicates that $\mathrm{Er}^{3+}$ ions are incorporated into different lattice locations depending on the Ga-flux.

More information on the optical activation of $\mathrm{Er}^{3+}$ in $\mathrm{GaN}$ can be drawn from power dependent PL studies under

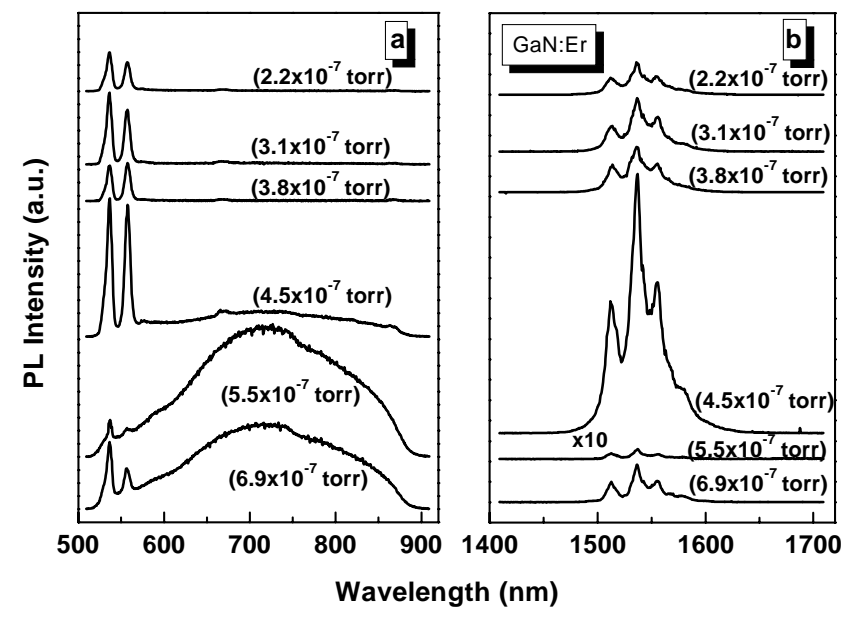

Fig. 1. Visible (a) and infrared (b) emission from Er-doped GaN as a function of Ga-flux under below-gap excitation $(496.5 \mathrm{~nm})$. For both cases, the $\mathrm{Er}^{3+}$ emission intensity reached its maximum under slightly N-rich flux near the stoichiometric growth condition (Ga-flux: $\sim 4.5 \times 10^{-7}$ Torr).

above-gap excitation (see Fig. 3). All investigated samples exhibited the onset of $1.54 \mu \mathrm{m}$ PL saturation at relatively low pump intensities $\left(<2 \mathrm{~W} / \mathrm{cm}^{2}\right)$, consistent with a high excitation efficiency for above-gap pumping [16]. At the same time, it can be noticed that the $\mathrm{Er}^{3+} \mathrm{PL}$ saturation level greatly varied for the different samples. It has been discussed in the literature before [16,22], that the $\mathrm{Er}^{3+} \mathrm{PL}$ saturation level is determined by the product of concentration of optically active $\mathrm{Er}$ ions $\left(N_{\mathrm{Er}}\right)$ and radiative decay rate $\left(w_{\mathrm{rad}}\right)$. PL lifetime studies revealed that $w_{\text {rad }}$ is approximately independent of Ga-flux. Therefore, the large difference in the PL saturation is attributed to a large difference in the concentration of optically active $\mathrm{Er}^{3+}$ ions. As can be derived from Fig. 3, the PL saturation level and hence $\mathrm{N}_{\mathrm{Er}}$ is $\sim 17$ times larger for the sample grown under slightly $\mathrm{N}$-rich con-

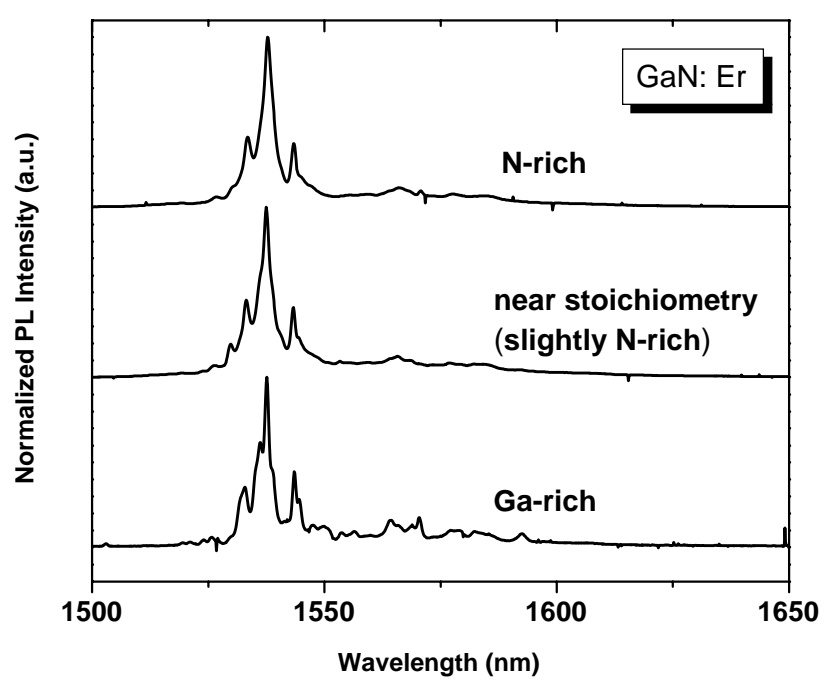

Fig. 2. High-resolution, infrared emission spectra at $15 \mathrm{~K}$ from Er-doped GaN grown under different Ga-fluxes. The emission was excited using below-gap excitation $(496.5 \mathrm{~nm})$. 


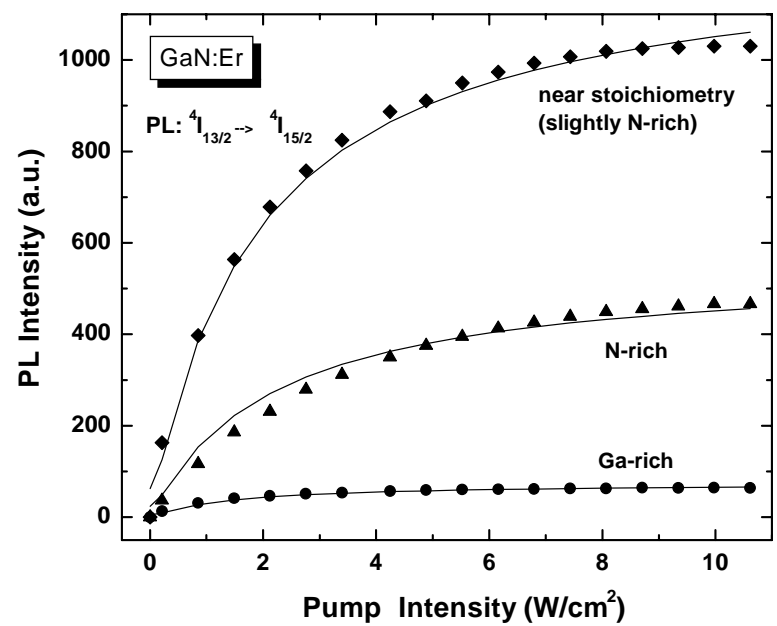

Fig. 3. Pump intensity dependence of the $1.54 \mu \mathrm{m}$ PL from GaN:Er samples grown with different Ga-flux during growth. The emission was excited using above-gap excitation $(336-363 \mathrm{~nm})$. The solid lines are guides for the eye.

ditions compared to that grown under Ga-rich conditions. In contrast, previous secondary ion mass spectroscopy (SIMS) studies have shown that the total Er concentration in the investigated GaN:Er films varied only by roughly a factor of two [18]. Therefore, the PL saturation result from Fig. 3 reveal that in GaN:Er grown under Ga-rich conditions only a small fraction $(<10 \%)$ of the total Er concentration is optically active under above-gap pumping. Similar observations were reported for Er-doped crystalline and amorphous $\mathrm{Si}$ [23]. More comparative PL saturation studies employing a well-characterized $\mathrm{Er}^{3+}$ sample (e.g. Er-doped $\mathrm{SiO}_{2}$ ) are required to determine the absolute concentration of optically active $\mathrm{Er}^{3+}$ ions in GaN:Er.

\subsection{Eu-doped GaN}

Eu-doped GaN is of great interest for display applications because of its bright red emission peaking around $622 \mathrm{~nm}$ $[14,24-28] . \mathrm{Eu}^{3+}$ is also known as a "spectroscopic probe" for the local environment of $\mathrm{Eu}^{3+}$ ions in solids, because the main emitting level is the non-degenerate ${ }^{5} \mathrm{D}_{0}$ state [20]. For example, observing the ${ }^{5} \mathrm{D}_{0} \leftrightarrow{ }^{7} \mathrm{~F}_{0}$ transition allows one to identify the number of emitting $\mathrm{Eu}^{3+}$ centers in a given host. Fig. 4 gives an overview of the low temperature emission of GaN:Eu. The main emission lines can be assigned to the transitions: ${ }^{5} \mathrm{D}_{0} \rightarrow{ }^{7} \mathrm{~F}_{5}(\sim 843 \mathrm{~nm}),{ }^{5} \mathrm{D}_{0} \rightarrow{ }^{7} \mathrm{~F}_{4}(\sim 715 \mathrm{~nm})$, ${ }^{5} \mathrm{D}_{0} \rightarrow{ }^{7} \mathrm{~F}_{3}(\sim 664 \mathrm{~nm}),{ }^{5} \mathrm{D}_{0} \rightarrow{ }^{7} \mathrm{~F}_{2}(\sim 622 \mathrm{~nm})$, and ${ }^{5} \mathrm{D}_{0} \rightarrow$ ${ }^{7} \mathrm{~F}_{1}(\sim 600 \mathrm{~nm})$. The weak emission features at $\sim 585$ and $\sim 590 \mathrm{~nm}$ are most likely due to ${ }^{5} \mathrm{D}_{0} \rightarrow{ }^{7} \mathrm{~F}_{0}$ transitions suggesting multiple $\mathrm{Eu}^{3+}$ centers in $\mathrm{GaN}[14,27,28]$.

In order to identify the ${ }^{5} \mathrm{D}_{0} \leftrightarrow{ }^{7} \mathrm{~F}_{0}$ transitions and associated $\mathrm{Eu}^{3+}$ centers more clearly, we have performed high-resolution PL excitation studies in the range from 560 to $595 \mathrm{~nm}$. The spectral resolution in the PLE measurements was limited by the linewidth of the employed excitation source $\left(\sim 0.2 \mathrm{~cm}^{-1}\right)$ (Fig. 5 ).

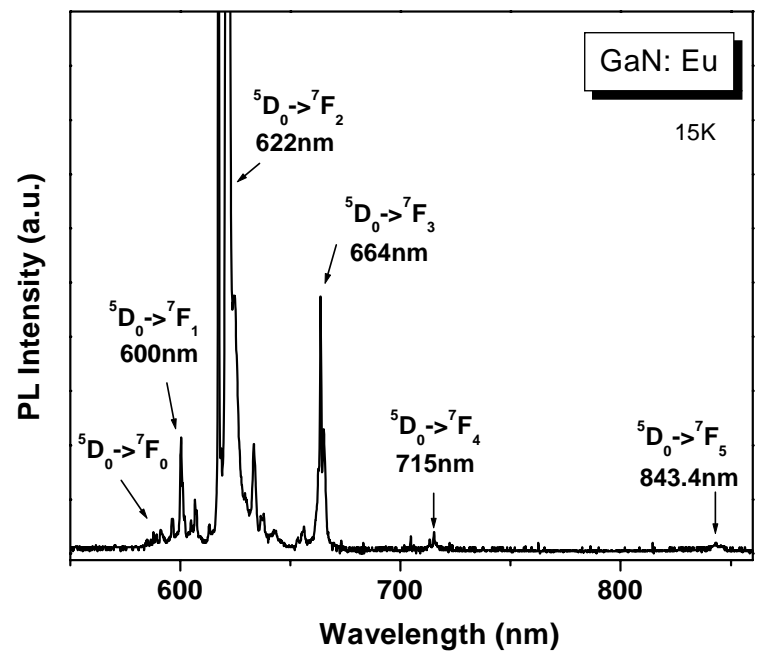

Fig. 4. Emission spectrum of $\mathrm{GaN}: \mathrm{Eu}$ at $15 \mathrm{~K}$ under above-gap excitation. The main emission features arising from the ${ }^{5} \mathrm{D}_{0}-{ }^{7} \mathrm{~F}_{\mathrm{J}}(J=0,1,2,3,4,5)$ transitions are indicated in the graph. More details on the identification of the ${ }^{5} \mathrm{D}_{0}-{ }^{7} \mathrm{~F}_{0}$ transitions are presented in Fig. 5.

At room temperature, four PLE peaks were observed at $571,585.2,588.8$, and $591.7 \mathrm{~nm}$. With decreasing temperature the peak at $591.7 \mathrm{~nm}$ reduced in intensity and was not observed at $15 \mathrm{~K}$. Therefore, this line does not originate from the ${ }^{7} \mathrm{~F}_{0}$ ground state, but is due to the thermal population of higher lying ${ }^{7} \mathrm{~F}_{1} /{ }^{7} \mathrm{~F}_{2}$ manifolds. The linewidth of all PLE peaks observed at room-temperature narrowed slightly with decreasing temperature and some fine structure was resolved. At low temperature (15 K) five PLE lines at 571, $585.8,587.9,588.9$, and $589.4 \mathrm{~nm}$ were observed and tentatively assigned to ${ }^{7} \mathrm{~F}_{0} \rightarrow{ }^{5} \mathrm{D}_{0}$ transitions. The ${ }^{7} \mathrm{~F}_{0} \rightarrow{ }^{5} \mathrm{D}_{0}$ transition at $\sim 571 \mathrm{~nm}$ is at an unusually low wavelength, but similar cases have been reported [29,30]. Therefore, we conclude that at least five different $\mathrm{Eu}^{3+}$ centers exist in GaN:Eu.

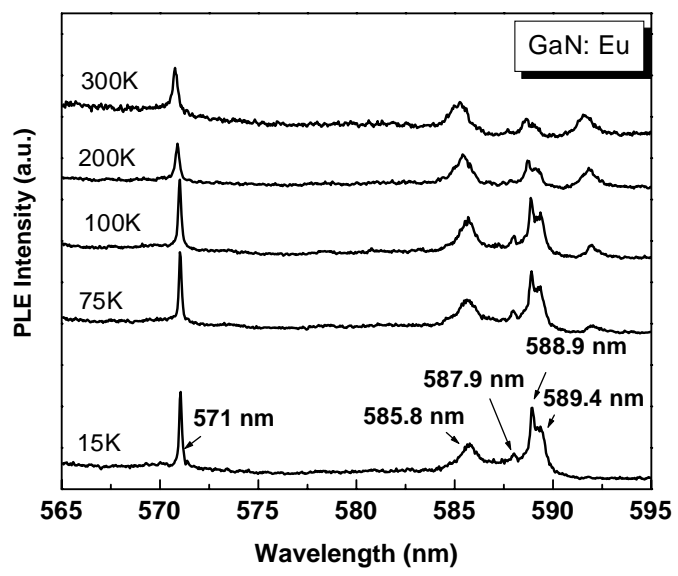

Fig. 5. High-resolution PLE spectrum of Eu-doped GaN at $15 \mathrm{~K}$. The emission was monitored at $\sim 623 \mathrm{~nm}$. Absorption lines located at 571, $585.5,587.9,588.9$, and $589.4 \mathrm{~nm}$ are tentatively assigned to the ${ }^{7} \mathrm{~F}_{0}-{ }^{5} \mathrm{D}_{0}$ transitions. 


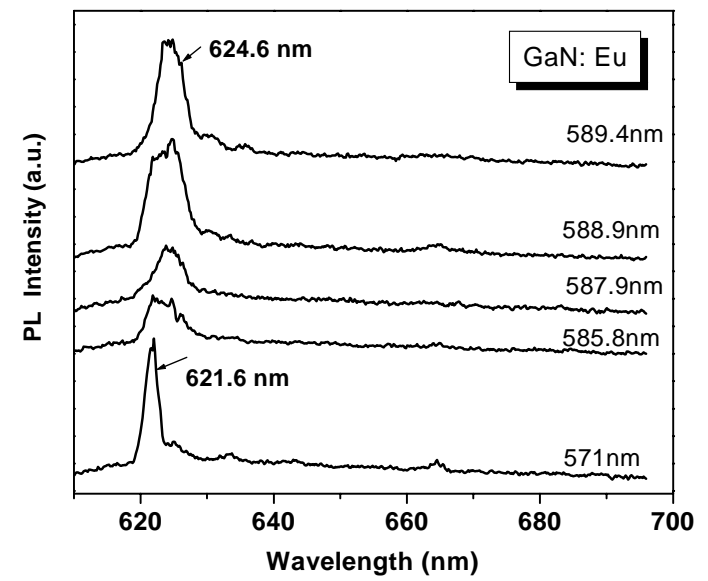

Fig. 6. Site-selective PL spectra at $15 \mathrm{~K}$ excited at 571, 585.5, 587.9, 588.9 , and $589.4 \mathrm{~nm}$. The $\mathrm{Eu}^{3+}$ center excited at $571 \mathrm{~nm}$ is distinct from the other $\mathrm{Eu}^{3+}$ centers and dominates under above-gap excitation.

More support for the identification of the different $\mathrm{Eu}^{3+}$ centers was obtained from site-selective PL measurements. Pumping resonantly into each of the PLE line revealed different $\mathrm{Eu}^{3+}$ emission spectra and lifetimes as shown in Fig. 6. The spectral differences are especially pronounced for the $\mathrm{Eu}^{3+}$ center excited at $571 \mathrm{~nm}$. This $\mathrm{Eu}^{3+}$ center exhibited a relatively narrow ${ }^{5} \mathrm{D}_{0} \rightarrow{ }^{7} \mathrm{~F}_{2} \mathrm{PL}$ at $\sim 622 \mathrm{~nm}$ with a nearly exponential lifetime of $\sim 235 \mu$ s. The ${ }^{5} \mathrm{D}_{0} \rightarrow{ }^{7} \mathrm{~F}_{2}$ PL spectra of the other $\mathrm{Eu}^{3+}$ centers are broader and shifted to longer wavelength $(\sim 624 \mathrm{~nm})$. In addition, the PL decay transients of these $\mathrm{Eu}^{3+}$ centers are non-exponential with average lifetimes of less than $200 \mu \mathrm{s}$. The smaller linewidth of the $571 \mathrm{~nm} \mathrm{Eu}^{3+}$ center indicates less inhomogeneous broadening compared to the other $\mathrm{Eu}^{3+}$ centers. It can be speculated that the $571 \mathrm{~nm}$ center is associated with $\mathrm{Eu}^{3+}$ ions in substitutional $\mathrm{Ga}^{3+}$ lattice positions, whereas the other $\mathrm{Eu}^{3+}$ centers are due to $\mathrm{Eu}^{3+}$ ions incorporated into interstitial sites with close vicinity to other defects and impurities. Interestingly, the PL spectrum excited at $571 \mathrm{~nm}$ is very similar to PL spectra obtained under above-gap pumping, which shows that this $\mathrm{Eu}^{3+}$ center dominates under carrier-mediated excitation. More site-selective PL and PLE studies of the different $\mathrm{Eu}^{3+}$ centers in $\mathrm{GaN}$ are in progress.

\subsection{Tm-doped $A l_{x} G a_{1-x} N(0 \leq x \leq 1)$}

PL spectra of Tm-doped GaN under above-gap excitation at 15,150 , and $300 \mathrm{~K}$ are shown in Fig. 7. The emission is dominated by a broad band centered at $\sim 530 \mathrm{~nm}$, which extends from roughly 450 to $750 \mathrm{~nm}$ and resembles the well-known yellow band emission from GaN [31,32]. An infrared emission line at $\sim 803 \mathrm{~nm}$ can be assigned to the intra-4f transition ${ }^{3} \mathrm{H}_{4} \rightarrow{ }^{3} \mathrm{H}_{6}$ of $\mathrm{Tm}^{3+}$ ions [33]. A weak shoulder at $\sim 479 \mathrm{~nm}$ indicates the blue emission line from the ${ }^{1} \mathrm{G}_{4} \rightarrow{ }^{3} \mathrm{H}_{6}$ transition of $\mathrm{Tm}^{3+}$. The PL intensity of $479 \mathrm{~nm}$ line increases slightly when cooling the sample down to $15 \mathrm{~K}$. Compared to the visible PL from $\mathrm{GaN}: \mathrm{Er}$

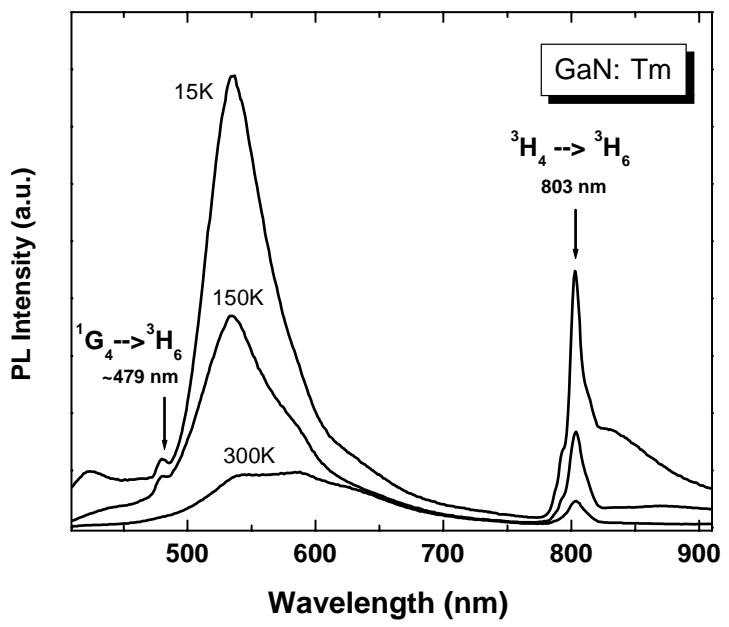

Fig. 7. PL spectra of Tm-doped $\mathrm{GaN}$ under above-gap excitation at 15 , 150 , and $300 \mathrm{~K}$. The shoulder at $479 \mathrm{~nm}$ indicates blue emission from the ${ }^{1} \mathrm{G}_{4} \rightarrow{ }^{3} \mathrm{H}_{6}$ intra-4f transition of $\mathrm{Tm}^{3+}$.

and $\mathrm{GaN}: \mathrm{Eu}$, the blue emission from GaN:Tm is orders of magnitude weaker under above-gap pumping, which indicates that $\mathrm{Tm}^{3+}$ ions are not efficiently excited through carrier recombination processes.

An energy transfer model based on a RE-related trap level was proposed by Takahei et al. for InP: $\mathrm{Yb}$ and later extended to other RE-doped semiconductors [34,35]. In this model, RE doping of a semiconductor leads to the formation of a RE related level in the band-gap of the host. This level can trap photo-excited free carriers, which subsequently recombine and transfer their energy to intra-4f RE transitions. Our recent PL excitation measurements of $\mathrm{GaN}: \mathrm{Eu}$ provided experimental evidence for an $\mathrm{Eu}$ trap-level at $\sim 0.3 \mathrm{eV}$ below the conduction band of $\mathrm{GaN}$ [27]. A similar level was reported by $\mathrm{Li}$ et al. using Fourier transform infrared (FTIR) measurements [9] and by Vantomme et al. using deep level transient spectroscopy (DLTS) [36]. An Er related trap-level at $\sim 0.3 \mathrm{eV}$ was also reported for $\mathrm{GaN}: \mathrm{Er}$ [37]. The recombination energy of carriers trapped at the $\mathrm{Eu} / \mathrm{Er}$ related traps in $\mathrm{GaN}$ is then estimated to be $\sim 3.1 \mathrm{eV}$, which energetically matches intra-4f transitions of $\mathrm{Eu}^{3+}$ and $\mathrm{Er}^{3+}$, respectively [19]. Therefore, the carrier-mediated energy transfer to $\mathrm{Er}^{3+}$ and $\mathrm{Eu}^{3+}$ seems to occur with high efficiency. On the contrary, the energy level structure of $\mathrm{Tm}^{3+}$ does not exhibit an energy level at $\sim 3.1 \mathrm{eV}$. Consequently, a carrier-mediated energy transfer process to $\mathrm{Tm}^{3+}$ ions is less likely to occur in GaN:Tm. A similar explanation was recently proposed for the poor above-gap excitation efficiency of $\mathrm{Tb}^{3+}$ ions in GaN:Tb [9].

In an effort to optimize the carrier-mediated excitation of $\mathrm{Tm}^{3+}$ ions in III-nitrides, we are currently exploring the energy transfer process to $\mathrm{Tm}^{3+}$ as a function of band-gap energy. Fig. 8 shows preliminary emission spectra for Tm-doped $\mathrm{Al}_{x} \mathrm{Ga}_{1-x} \mathrm{~N}$ with $x=0(\mathrm{GaN}), 0.13,0.24,0.29$, 0.44 , and $1(\mathrm{AlN})$. The corresponding band-gap energies are $3.4 \mathrm{eV}(\mathrm{GaN}), 3.64,3.88,3.99,4.89$, and $6.2 \mathrm{eV}(\mathrm{AlN})$ 


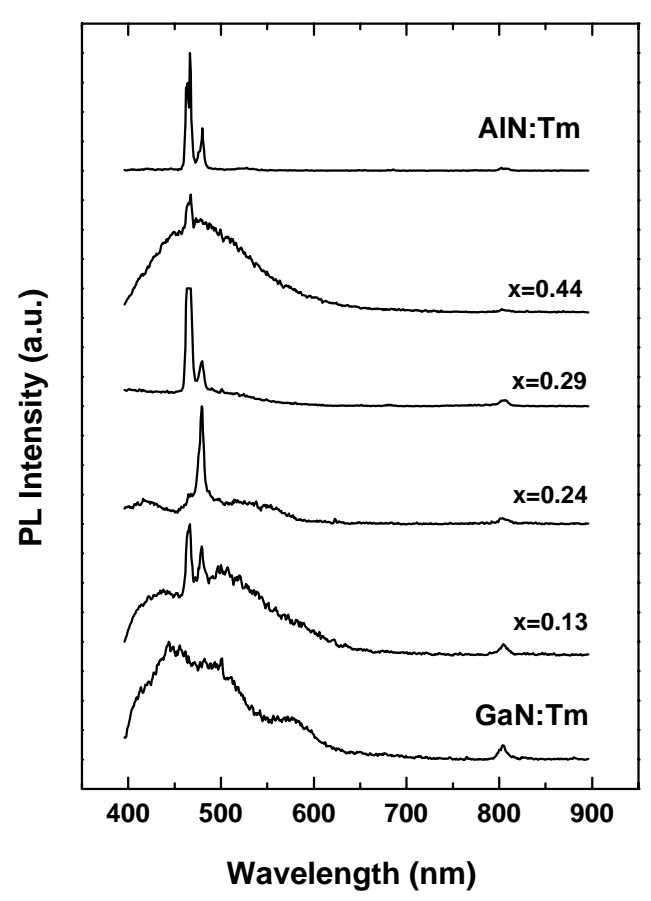

Fig. 8. PL spectra of Tm-doped $\mathrm{Al}_{x} \mathrm{Ga}_{1-x} \mathrm{~N}$ with $x=0(\mathrm{GaN}), 0.13$, $0.24,0.29,0.44$, and $1(\mathrm{AlN})$ under $250 \mathrm{~nm}$ excitation.

respectively. The emission was excited at $250 \mathrm{~nm}$, which corresponds to above-gap pumping for all investigated AlGaN samples, but results in below-gap excitation for AlN:Tm. As discussed before, hardly any blue emission was observed from GaN:Tm (lowest trace in Fig. 8). With increasing $\mathrm{Al}$ content an enhancement of the $478 \mathrm{~nm}$ line from $\mathrm{Tm}^{3+}$ is observed. In addition, a new blue emission line appears at $\sim 466 \mathrm{~nm}$. Based on a comparison to existing literature [38], the $466 \mathrm{~nm}$ line is tentatively attributed to emission from the ${ }^{1} \mathrm{D}_{2} \rightarrow{ }^{3} \mathrm{~F}_{4}$ transition of $\mathrm{Tm}^{3+}$ ions. The overall blue emission from both lines at 466 and $479 \mathrm{~nm}$ reached a maximum for an $\mathrm{Al}$ content of $x=0.29$, with the $466 \mathrm{~nm}$ line having the highest PL intensity. At higher $\mathrm{Al}$ content the total blue emission started to decrease and at $x=0.44$ only weak blue emission at $466 \mathrm{~nm}$ was observed. On the contrary, below-gap pumping at $250 \mathrm{~nm}$ of AlN:Tm resulted into strong blue emission with intense lines at $\sim 466$ and $479 \mathrm{~nm}$. For AlN:Tm, the blue emission is most likely excited through some broad defect level in the host.

Fig. 8 clearly demonstrates the sensitivity of the blue $\mathrm{Tm}^{3+}$ emission on the $\mathrm{Al}$ content and hence the band-gap of AlGaN. It cannot be excluded, however, that also chemical effects related to the presence of $\mathrm{Al}$ change the $\mathrm{Tm}^{3+}$ incorporation and excitation mechanisms, similar to observations made for Er-doped AlGaAs $[39,40]$. The initial spectroscopic data on $\mathrm{Al}_{x} \mathrm{Ga}_{1-x} \mathrm{~N}$ :Tm indicate that the excitation efficiency of the ${ }^{1} \mathrm{G}_{4}$ state of $\mathrm{Tm}^{3+}$ increases up to $x \sim 0.24$. In addition, the larger bandgap of AlGaN compared to $\mathrm{GaN}$ moves the ${ }^{1} \mathrm{D}_{2}$ level of $\mathrm{Tm}^{3+}$ within the band-gap of the host, which results in emission at $466 \mathrm{~nm}$. A more detailed analysis of the emission properties of AlGaN:Tm and AlN:Tm is still in progress and will be discussed in a forthcoming paper.

\section{Conclusions}

Spectroscopic results on the PL properties of GaN:Er, GaN:Eu, GaN:Tm and AlGaN:Tm were presented. High-resolution PL and pump-power dependent PL studies of $\mathrm{GaN}$ :Er samples revealed that the $\mathrm{Er}^{3+}$ incorporation and the concentration of optically active Er ions is strongly dependent on the Ga-flux during MBE growth. Based on PL saturation experiments, we concluded that only a small fraction $(<10 \%)$ of the total $\mathrm{Er}$ ions in $\mathrm{GaN}$ are optically active for samples grown under Ga-rich conditions. More PL saturation experiments are still in progress to determine the absolute concentration of optically active $\mathrm{Er}^{3+}$ ions in $\mathrm{GaN}$ :Er films. The low optical activation of $\mathrm{Er}^{3+}$ ions in $\mathrm{GaN}$ is similar to recent observations made for Er-doped $\mathrm{Si} /$ amorphous $\mathrm{Si}$ [23]. This issue needs to be further addressed in future investigations to explore the full potential of RE-doped GaN for device applications. High-resolution PL excitation studies were performed on GaN:Eu and allowed the identification of at least five $\mathrm{Eu}^{3+}$ centers. An absorption line at an unusually low wavelength of $\sim 571 \mathrm{~nm}$ was also tentatively assigned to the ${ }^{5} \mathrm{D}_{0} \leftrightarrow{ }^{7} \mathrm{~F}_{0}$ transition. The $\mathrm{Eu}^{3+}$ center selectively excited through this $571 \mathrm{~nm}$ absorption line is distinct from the other $\mathrm{Eu}^{3+}$ centers and seems to dominate the above-gap pumped PL emission spectrum. GaN:Tm exhibited only a weak blue emission from the ${ }^{1} \mathrm{G}_{4} \rightarrow{ }^{3} \mathrm{H}_{6}$ transition of $\mathrm{Tm}^{3+}$ ions. A significant enhancement of the blue $\mathrm{Tm}^{3+}$ emission was obtained under above-gap pumping of Tm-doped $\mathrm{Al}_{x} \mathrm{Ga}_{1-x} \mathrm{~N}$ samples. Besides emission from the ${ }^{1} \mathrm{G}_{4} \rightarrow{ }^{3} \mathrm{H}_{6}$ transition, a second blue emission line appeared around $466 \mathrm{~nm}$ for $x>0.13$, which was assigned to the ${ }^{1} \mathrm{D}_{2} \rightarrow{ }^{3} \mathrm{~F}_{4}$ transition of $\mathrm{Tm}^{3+}$. Strong blue emission from the ${ }^{1} \mathrm{D}_{2}$ and ${ }^{1} \mathrm{G}_{4}$ levels of $\mathrm{Tm}^{3+}$ was also observed from Tm-doped AlN under below-gap excitation. The large sensitivity of the blue emission from $\mathrm{Tm}^{3+}$ on the band-gap of AlGaN suggests the possibility to optimize the RE excitation and emission properties through careful band-gap engineering of the host.

\section{Acknowledgements}

The authors from H.U. acknowledge financial support by ARO through grant DAAD19-02-1-0316. The work at U.C. was supported by ARO grant DAAD19-99-1-0348. Helpful discussions with F. Pelle and F. Auzel are also acknowledged.

\section{References}

[1] A.J. Steckl, J.M. Zavada, MRS Bull. 24 (9) (1999) 33-38.

[2] A.J. Steckl, J.C. Heikenfeld, D.S. Lee, M.J. Garter, C.C. Baker, Y. Wang, R. Jones, IEEE J. Sel. Top. Quant. 8 (2002) 749. 
[3] S. Morishima, T. Maruyama, M. Tanaka, Y. Masumoto, K. Akimoto, Phys. Stat. Sol. A 176 (1999) 113.

[4] V.I. Dimitrova, P.G. Van Patten, H.H. Richardson, M.E. Kordesh, Appl. Phys. Lett. 77 (2000) 478.

[5] Rare earth doped semiconductors. I, in: G.S. Pomerenke, P.B. Klein, D.W. Langer (Eds.), Proceedings of the Materials Research Society, Vol. 301, 1993.

[6] Rare earth doped semiconductors. II, in: S. Coffa, A. Polman, R.N. Schwartz, (Eds.), Proceedings of the Materials Research Society, Vol. 422, 1996

[7] Rare earth doped semiconductors. III, in: J. Zavada, T. Gregorkiewicz, A.J. Steckl (Eds.), Proceedings of E-MRS Symposium Spring 2000, Mater. Sci. Eng. B 81 (2001).

[8] D.S. Lee, J. Heikenfeld, A.J. Steckl, U. Hömmerich, J.T. Seo, A. Braud, J.M. Zavada, Appl. Phys. Lett. 79 (2001) 719.

[9] Z. Li, H. Bang, G. Piao, J. Sawahata, K. Akimoto, J. Cryst. Growth 240 (2002) 382

[10] J.T. Torvik, C.H. Qui, R.J. Feuerstein, J.I. Pankove, F. Namavar, J. Appl. Phys. 81 (1997) 6343.

[11] H.J. Lozykowski, W.M. Jadwisienczak, I.G. Brown, Appl. Phys. Lett. 74 (1999) 1129.

[12] E. Alves, M.F. da Silva, J.C. Soares, R. Vianden, J. Bartels, A. Kozanecki, Nucl. Instrum. Methods B 147 (1999) 383.

[13] S. Kim, S.J. Rhee, X. Li, J.J. Coleman, S.G. Bishop, P.B. Klein, J. Electron. Mater. 27 (1998) 246.

[14] T. Monteiro, C. Boemare, M.J. Soares, R.A. Sa Ferreira, L.D. Carlos, K. Lorenz, R. Vianden, E. Alves, Physica B 308-310 (2001) 22.

[15] U. Wahl, A. Vantomme, G. Langouche, J.P. Araujo, L. Peralta, J.G. Correia,. The ISOLDE collaboration J. Appl. Phys. 88 (2000) 1319.

[16] J.T. Seo, U. Hömmerich, D.S. Lee, J. Heikenfeld, A.J. Steckl, J.M. Zavada, J. Alloys Comp. 342 (2002) 62.

[17] A. Steckl, R. Birkhahn, Appl. Phys. Lett. 73 (1998) 1700.

[18] D.S. Lee, A.J. Steckl, Appl. Phys. Lett. 80 (2002) 728.

[19] G.H. Dieke, Spectra and Energy Levels of Rare Earth Ions in Crystals, Wiley, New York, 1968.

[20] B. Henderson, G.F. Imbusch, Optical Spectroscopy of Inorganic Solids, Clarendon Press, Oxford, UK, 1989.

[21] E. Calleja, M.A. Sanchez-Garcia, F.J. Sanchez, F. Calle, F.B. Naranjo, E. Munoz, U. Jahn, K. Ploog, Phys. Rev. B 62 (2000) 16826.
[22] F. Priolo, G. Franzo, S. Coffa, A. Carnera, Phys. Rev. B. 57 (1998) 4443.

[23] O.B. Gusev, M.S. Bresler, P.E. Pak, I.N. Yassievich, M. Forcales, N.Q. Vinh, T. Gregorkiewicz, Phys. Rev. B 64 (2001) 075302.

[24] J. Heikenfeld, M. Garter, D.S. Lee, R. Birkhahn, A.J. Steckl, Appl. Phys. Lett. 75 (1999) 1189.

[25] H.J. Lozykowski, W.M. Jadwisienczak, J. Han, I.G. Brown, Appl. Phys. Lett. 77 (2000) 767.

[26] M. Overberg, K.N. Lee, C.R. Abernathy, S.J. Pearton, W.S. Hobson, R.G. Wilson, J.M. Zavada, Mater. Sci. Eng. B 81 (2001) 150.

[27] E.E. Nyein, U. Hommerich, J. Heikenfeld, D.S. Lee, A.J. Steckl, J.M. Zavada, Appl. Phys. Lett. 82 (2003) 1655.

[28] H. Bang, S. Morishima, Z. Li, K. Akimoto, M. Nomura, E. Yagi, Phys. Stat. Sol. B 228 (2001) 319.

[29] R. Ternane, G. Panczer, M.Th. Cohen-Adad, C. Goutaudier, G. Boulon, N. Kbir-Ariguib, M. Trabesli-Ayedi, Opt. Mater. 16 (2001) 291.

[30] E. Antic-Fidancev, J. Alloys Comp. 300301 (2002) 2.

[31] T. Ogino, M. Aoki, Jpn. J. Appl. Phys. 19 (1980) 2395.

[32] S.J. Pearton, J.C. Zolper, R.J. Shul, F. Ren, J. Appl. Phys. 86 (1999) 1.

[33] D.S. Lee, A.J. Steckl, Appl. Phys. Lett. 82 (2003) 55

[34] K. Takahei, A. Taguchi, H. Nakagome, K. Uwai, P.S. Whitney, J. Appl. Phys. 66 (1989) 4941.

[35] A. Taguchi, K. Takahei, J. Appl. Phys. 79 (1996) 4330.

[36] A. Vantomme, B. Pipeleers, V. Matias, E. Alves, K. Lorenz, R.W. Martin, S. Dalmasso, K.P. O'Donnell, A. Braud, J.L. Doualan, European Materials Society Spring Meeting 2003, Strasbourg, France, paper J-VI.1.

[37] S. Kim, S.J. Rhee, X. Li, J.J. Coleman, S.G. Bishop, P.B. Klein, J. Electron. Mater. 27 (1998) 246.

[38] J.B. Gruber, A.O. Wright, M.D. Selter, B. Zandi, L.D. Merkle, J.A Hutchinson, C.A. Morrison, T.H. Allik, B.H.T. Chai, J. Appl. Phys. 81 (1997) 6585.

[39] T. Zhang, J. Sun, N.V. Edwards, D.E. Moxey, R.M. Kolbas, P.J. Caldwell, Mater. Res. Soc. Symp. Proc. 301 (1993) 257.

[40] T.D. Culp, U. Hömmerich, J.M. Redwing, T.F. Kuech, K.L. Bray, J. Appl. Phys. 82 (1997) 368. 